Invest. pens. crit.

Vol. 2, No. 3, enero-abril 2014.

pp. $35-52$

\title{
Prevalencia estimada de trastornos conductuales y emocionales en la población pediátrica en Panamá
}

\section{Velarde Herrera, S. ${ }^{1,2}$ Sánchez-Gómez, E. ${ }^{2}$ Manzané, F. 3,4 y Britton, G. ${ }^{4,5, *}$}

${ }^{1}$ Hospital de Especialidades Pediátricas de la Caja del Seguro Social. Panamá, Rep. de Panamá ${ }^{2}$ Clínica Neuropsicológica. Panamá, Rep. de Panamá

3Facultad de Psicología, Universidad Nacional de Panamá. Panamá, Rep. de Panamá

${ }^{4}$ Centro de Neurociencias y Unidad de Investigación Clínica, Instituto de Investigaciones Científicas y Servicios de Alta Tecnología (INDICASAT AIP). Apartado Postal 0843-01103, Ciudad del Saber, Panamá, Rep. de Panamá

${ }^{5}$ Escuela de Psicología, Universidad Santa María la Antigua, Apartado Postal 0819-08550, Panamá, Rep. de Panamá

* Autor para correspondencia: Email: gbritton@indicasat.org.pa

Recibido: 21 de febrero de 2014

Aceptado: 15 de abril de 2014

\begin{abstract}
This study intended to estimate the prevalence of behavioral and emotional disorders in a sample of school-aged children in the city of Panama through cross-sectional analyses conducted during the 2009 academic year. We selected a random sample of students of both sexes between the ages of 6 and 11 years from the eastern sector of the Metropolitan Region of Education. We employed The BASC-2 Structured Developmental History, a comprehensive history and background survey that provides an overview of social, psychological, developmental, educational, and health information about the child. The scale items are related to diagnostic categories of the Diagnostic and Statistical Manual of Mental Disorders (DSM-IV) and data are obtained through parent and teacher interviews. A total of 131 dyads of parents and teachers were included in the analysis. The estimated global prevalence was $8.1 \%$. The analysis of individual disorders indicated that the most prevalent conditions were learning difficulties (20.6\%), poor study skills $(26.0 \%)$ and anxiety $(15.3 \%)$ according to teachers, whereas parents rated withdrawal $(10.7 \%)$, anxiety $(8.4 \%)$ and lack of leadership $(6.9 \%)$ as most prevalent. Less than
\end{abstract}


Invest. pens. crit.

Vol. 2, No. 3, enero-abril 2014.

pp. $35-52$

one in ten children with a disorder was reported as having had contact with mental health services. This study is the first to attempt to estimate the prevalence of psychiatric disorders in the pediatric population of Panama. The data indicate an overall prevalence of disorders within the lower range of rates reported in previous studies conducted in Latin America. However, a notable number of children were rated as having learning- and anxiety-related problems, which underscores the need to evaluate further the mental health needs in pediatric populations in future studies.

Keywords: Child psychiatry; Primary education; Learning; Cognition; Behavioral assessment

\section{Resumen}

Objetivo. Estimar la prevalencia de trastornos conductuales y emocionales en una muestra de niños de edad escolar de la ciudad de Panamá mediante un estudio transversal realizado en el año escolar 2009.

Métodos. Se obtuvo una muestra al azar de estudiantes de ambos sexos entre las edades de 6 y 11 años del sector este de la Región Metropolitana de Educación. Se utilizó un instrumento de evaluación estandarizado (BASC) diseñado para facilitar el diagnóstico de la clasificación pedagógica de una variedad de trastornos. La escala evalúa diversos aspectos de la conducta y la personalidad de los niños relacionados con los criterios diagnósticos de categorías de psicopatología del Manual Diagnóstico y Estadístico de los Trastornos Mentales (DSM-IV). La escala se aplica mediante cuestionarios suministrados a padres y maestros.

Resultados. Un total de 131 díadas de padres y maestros fueron incluidas en los análisis. La prevalencia global estimada fue de $8.1 \%$. El análisis de los trastornos individuales indicó que las condiciones más prevalentes fueron los problemas de aprendizaje $(20.6 \%)$, las habilidades de estudio $(26.0 \%)$ y la ansiedad (15.3\%) según los maestros. Según los padres, las condiciones más prevalentes fueron el aislamiento (10.7\%), la ansiedad (8.4\%) y la falta de liderazgo (6.9\%). Menos de uno en diez de los niños con un trastorno tuvo contacto con servicios de salud mental.

Conclusiones. Este estudio es el primero que intenta estimar la prevalencia de trastornos psiquiátricos en la población pediátrica en Panamá. Los datos del estudio muestran una prevalencia de trastornos dentro del límite inferior a las cifras reportadas en estudios previos realizados en Latinoamérica. Sin embargo, un número considerable de niños mostró problemas de ansiedad y escolares, lo que destaca la importancia de evaluar las necesidades en salud mental en poblaciones pediátricas en estudios posteriores. 
Invest. pens. crit.

Vol. 2, No. 3, enero-abril 2014.

pp. $35-52$

Palabras clave: Psiquiatría infantil; Educación primaria; Aprendizaje; Cognición; Evaluación conductual

\section{Introducción}

Existe una preocupación a nivel mundial en cuanto a la frecuencia de los trastornos mentales en la población general, y los niños forman uno de los segmentos de la población más vulnerables. En la última década ha surgido evidencia epidemiológica sobre la alta prevalencia de trastornos mentales en niños y adolescentes $(1,2,3)$, la cual, cuando se compara con la prevalencia en adultos, resalta la importancia de la intervención temprana sobre la salud mental en la adultez $(4,5)$. Por esta razón, organizaciones internacionales reiteran la necesidad de realizar estudios para recopilar los datos esenciales sobre los sistemas nacionales de salud mental, y así establecer planes estratégicos dirigidos a disminuir la discapacidad asociada a los trastornos mentales (6).

Algunos miembros de la comunidad científica como el editor de la revista Nature han denominado a los siguientes diez años "La década de los trastornos mentales" (7) y en la actualidad se promueven una serie de eventos académicos para reforzar este concepto. En Panamá se realizó la Conferencia Regional de Salud Mental en octubre de 2010 para evaluar el camino recorrido por la región y los logros en cuanto a políticas y servicios de Salud Mental propuestos en la Declaración de Caracas $(8,9)$. Los retos resaltados incluyen factores sociales, económicos y políticos, agravados por la escasez de estudios epidemiológicos necesarios para el desarrollo de políticas adecuadas de salud mental. La falta de atención a la investigación en salud mental es problemática debido a que se estima que una de cada cuatro personas tendrá un trastorno mental en algún momento de su vida (6).

El objetivo de identificar las necesidades en la salud mental poblacional es especialmente vital cuando se trata de personas en pleno crecimiento y desarrollo neural como es el caso de la población pediátrica. Este grupo etario sigue siendo un grupo extremadamente vulnerable debido a la amplia gama de factores dinámicos que caracterizan el desarrollo neural $(10,11)$ y los efectos a largo plazo asociados a la enfermedad mental durante el desarrollo temprano (3). Otros aspectos de índole socioeconómica son también agentes causales de patología neuropsiquiátrica. Estudios con modelos animales y humanos han demostrado la influencia que tienen los factores de estrés crónico sobre el desarrollo neural y las funciones cognitivas (12,13). Estos sugieren que la tasa de problemas mentales puede ser mucho mayor en niños que viven en condiciones de pobreza, inestabilidad, y abandono. Este punto tiene una alta relevancia en países de Latinoamérica donde los menores de 15 años y aquellos que viven en la pobreza constituyen una proporción significativa de la población (14).

Existen iniciativas para mejorar el conocimiento de las condiciones neuropsiquiátricas a nivel internacional, como el Proyecto ATLAS que tiene como objetivo conocer la realidad de los recursos disponibles y los sistemas de salud mental para niños y adolescentes a nivel mundial 
Invest. pens. crit.

Vol. 2, No. 3, enero-abril 2014.

pp. $35-52$

(15). La intención de recabar datos demográficos e información sobre políticas legislativas, recursos humanos, disponibilidad y uso de medicamentos en el tema de salud mental es la base para el diseño de planes que se apoyan en las realidades de cada país. Lamentablemente Panamá no aparece en la lista de los países que han completado el cuestionario ATLAS y en general, en América Latina, salvo algunas excepciones, la información relacionada a los sistemas de salud mental es escasa (3).

Avances en las estandarizaciones de definiciones operativas para los trastornos psiquiátricos utilizando la Clasificación Internacional de Enfermedades (CIE-10) (16) y el Manual Diagnóstico y Estadístico de los Trastornos Mentales (DSM IV)(17) han mejorado el diagnóstico de trastornos psiquiátricos, pero aún existe la necesidad de mejorar los criterios diagnósticos, particularmente cuando se trata de pacientes pediátricos (18). Como ejemplo, las cifras de prevalencia de trastornos mentales en niños y adolescentes oscilan entre 1 y 51\% (19), lo que se asocia a diferencias en el tipo de muestra examinado, ya sea una muestra de comunidad o clínica, y a la variabilidad de los instrumentos utilizados (20-22). En Latinoamérica se han efectuado revisiones del tema mediante exploraciones de los artículos publicados en los anteriores 10 años y coinciden en que 1 de cada 5 niños presenta una condición psiquiátrica $(1,23)$.

Se estima que la prevalencia de los trastornos psiquiátricos en los adultos oscila entre 15 y $20 \%$ (24), y existe una tendencia general hacia un aumento en estas cifras. Reportes de los años 90 señalaban una pérdida de años de vida ajustados a la discapacidad debido a condiciones neuropsiquiátricas de 8.8\% que aumentó a 22.2\% en 2002 (25). Por lo tanto, se espera que en los próximos años incrementará la demanda en la atención y tratamiento de trastornos mentales $(21,26)$, lo que hace necesaria la identificación de problemas lo más temprano posible para implementar oportunamente las medidas preventivas (27).

El presente estudio es el primero en Panamá que intenta estimar la prevalencia de trastornos psiquiátricos en una muestra poblacional urbana de niños entre 6 y 11 años utilizando una escala de evaluación estandarizada para la evaluación de psicopatología y la cuantificación de constructos asociados a trastornos mentales. En la última década, el uso de dichas escalas ha incrementado la objetividad de evaluaciones de trastornos en niños de edad escolar ya que toman en cuenta diferentes fuentes de información del hogar y el colegio $(28,29)$. Aunque las escalas no deben sustituir el diagnóstico clínico, son utilizadas ampliamente en la investigación y la práctica como evaluación preliminar de trastornos mentales y muestran alta correlación con el diagnóstico del DSM IV (29).

\section{Materiales y métodos}

\section{Selección de la muestra}

Este estudio transversal se realizó en dos colegios públicos ubicados en el sector este de la región metropolitana de educación de la ciudad de Panamá. Ambos colegios cuentan con una tasa de 
Invest. pens. crit.

Vol. 2, No. 3, enero-abril 2014.

pp. $35-52$

matrícula en el quintil superior de acuerdo a los registros nacionales del Ministerio de Educación. Las viviendas en el sector este del distrito municipal reflejan la diversidad demográfica de niveles socioeconómicos bajo y medio-bajo de acuerdo al censo nacional (30). El estudio se realizó entre marzo y diciembre 2009, un período que abarca un año escolar. A inicios del año escolar se enviaron invitaciones a padres de familia a participar en un estudio cuyo objetivo era evaluar factores asociados con el aprendizaje y la conducta en niños de edad escolar. Aproximadamente 91\% de los padres aceptaron participar, y 242 niños fueron seleccionados al azar. La muestra constituye $0.51 \%$ de los estudiantes matriculados en colegios públicos a nivel primaria en el distrito municipal en el 2009.

\section{Procedimiento de evaluación}

El protocolo fue aprobado por el comité científico y de bioética del INDICASAT AIP y recibió el aval del Ministerio de Educación. Todas las evaluaciones fueron realizadas por evaluadores idóneos y tuvieron lugar dentro de las escuelas. El/la padre/madre o cuidador principal del niño fue entrevistado durante el primer trimestre del año escolar; el maestro fue entrevistado durante el último trimestre. Se obtuvo consentimiento informado de todos los padres de familia al momento del primer contacto. Se utilizó la Escala Multidimensional de la Conducta (BASC, del inglés Behavior Assessment System for Children) (31) para estimar la prevalencia de problemas conductuales y emocionales en la muestra.

El BASC es un instrumento estandarizado en poblaciones hispanas en los Estados Unidos y en ámbitos latinoamericanos para la identificación de trastornos de conducta, trastornos emocionales, y dificultades escolares en niños escolares y en la clínica (32,33). El BASC sirve para evaluar a niños desde los 2 años 6 meses hasta los 18 años y mide conductas a lo largo de dimensiones clínicas y adaptativas. Los cuestionarios requieren que un padre/cuidador y un maestro determinen la frecuencia de diversas conductas observables durante los 6 meses antes de la evaluación, lo que obedece al conocimiento que algunos comportamientos se expresan en diferentes grados en el hogar y el colegio y pueden ser detectados mejor por un informante que por otro (29). El uso del BASC en la investigación ha logrado establecer su adaptación a la región, su alto valor interpretativo, consistencia interna (coeficientes alfa entre .85-.95), y facilidad para administrar y calificar $(28,34,35)$.El BASC muestra alta correlación (0.72-0.84) con otros instrumentos similares, lo que sustenta la validez de sus constructos $(28,31)$.

El BASC consiste de 9 escalas clínicas y 5 escalas adaptativas. Cada escala arroja un puntaje $T$ (promedio $=50$, desviación estándar $=10)$. Puntajes en las escalas clínicas a más de 1 desviación estándar (DE) por arriba de la media $(T=60)$ se consideran indicadores de "riesgo"; puntajes 2 DE por arriba de la media $(T=70)$ son clínicamente significativos. En las escalas adaptativas, puntajes a más de $1 \mathrm{DE}$ por debajo de la media $(T=40)$ se consideran indicadores de "riesgo"; puntajes $2 \mathrm{DE}$ por debajo de la media $(T=30)$ indican bajo funcionamiento. La calificación de la prueba se realiza mediante un software del BASC que brinda además índices de validez que 
Invest. pens. crit.

Vol. 2, No. 3, enero-abril 2014.

pp. $35-52$

permiten determinar la veracidad y consistencia de los informantes. Los aspectos cualitativos que definen cada concepto dentro de las diferentes escalas clínicas y adaptativas de los cuestionarios son los siguientes (31):

Escalas clínicas

- Hiperactividad: Tendencia a ser excesivamente activo y actuar sin pensar.

- Agresión: Tendencia a actuar de manera hostil (verbal o físicamente).

- Problemas de conducta: Tendencia a actuar de manera antisocial.

- Depresión: Sentimientos de infelicidad y estrés que pueden interferir con las actividades cotidianas (síntomas neurovegetativos).

- Somatización: Tendencia a quejarse de pequeñas dolencias físicas.

- Ansiedad: Tendencia al nerviosismo y la preocupación.

- Problemas de atención: Tendencia a distraerse fácilmente y dificultad en mantener la concentración.

- Problemas de aprendizaje: Presencia de dificultades en el estudio, particularmente en la comprensión o realización de trabajo escolar (solo en cuestionario de maestros).

- Atipicidad: Tendencia a manifestar conducta inusual comúnmente asociada con psicosis (como por ejemplo, alucinaciones visuales o auditivas).

- Aislamiento: Tendencia a evitar contacto social.

Escalas adaptativas

- Adaptabilidad: Habilidad para adaptarse a cambios ambientales.

- Liderazgo: Habilidades asociadas con la consecución de metas académicas, en particular, habilidad de trabajar bien con los demás.

- Habilidades sociales: Habilidades necesarias para interactuar eficazmente con compañeros y adultos en el hogar, la escuela y la comunidad.

- Habilidades en el estudio: Habilidades que conducen a resultados académicos positivos, incluyendo habilidad organizativa y buenos hábitos de estudio (solo en cuestionario de maestros).

En el diagnóstico de trastornos pediátricos comprendidos en el DSM IV existe un consenso en cuanto a la importancia de obtener los aportes de múltiples informantes (ej. padres y maestros) para integrar información sobre el niño en diversos contextos y situaciones $(28,29)$. Por lo tanto, para la estimación de prevalencias en el presente estudio sólo los niños cuyos padres y maestros conjuntamente obtuvieron resultados de veracidad y consistencia aceptables en los cuestionarios fueron incluidos en los análisis, lo que nos permitió examinar también el grado de correlación entre los informantes. Además, en base a estudios anteriores que mostraron diferencias entre niños y niñas en la prevalencia de diferentes trastornos psiquiátricos $(2,14,36)$, se evaluó la 
Invest. pens. crit.

Vol. 2, No. 3, enero-abril 2014.

pp. $35-52$

prevalencia de trastornos y su relación con el género. El análisis estadístico se efectuó mediante la aplicación de la prueba Chi cuadrado para frecuencias y porcentajes, y el coeficiente Pearson para evaluar el grado de correlación entre informantes.

\section{Resultados}

De las 242 díadas de cuestionarios BASC aplicados, 13 (5.4\%) fueron excluidas debido a una clasificación incorrecta en los registros escolares y 98 (40.5\%) no cumplieron con el criterio de obtener índices de veracidad y consistencia aceptables para ambos informantes $(90.1 \%$ y 59.9\% de los cuestionarios de maestros y padres, respectivamente, resultaron en índices aceptables). La muestra final consistió de 131 niños con díadas de cuestionarios que fueron incluidas en el estimado de prevalencias. Las características demográficas de la muestra están resumidas en la Tabla 1. La prevalencia total de los trastornos del BASC fue $8.1 \%$. De los 15 niños que recibieron algún tipo de atención en servicios de salud mental a lo largo del desarrollo (ej. psiquiátrica, psicológica, neurológica), $12(9.2 \%)$ tuvieron al menos 1 trastorno de acuerdo a los padres y maestros. Ningún niño había recibido fármacos neuropsiquiátricos por más de 6 meses en su vida.

Los puntajes promedio en cada escala de los BASC padres y maestros están resumidos en la Tabla 2. Las prevalencias estimadas, basadas en puntajes en rangos de riesgo, bajo funcionamiento y clínicamente significativos, están resumidas en la Tabla 3. Los maestros (12.3\%) reportaron una prevalencia total de trastornos mayor que los padres (3.9\%). Los padres y maestros estuvieron de acuerdo en cuanto a los problemas de atención, problemas de conducta, agresión e hiperactividad, aunque el grado de correlación fue bajo (ver Tabla 2); sin embargo, las demás correlaciones fueron menores de 0.1 y no significativas. Las condiciones más prevalentes fueron las habilidades de estudio (26.0\%), los problemas de aprendizaje $(20.6 \%)$ y la ansiedad (15.3\%) según los maestros. Según los padres las condiciones más prevalentes fueron el aislamiento $(10.7 \%)$, la ansiedad (8.4\%) y la falta de liderazgo (6.9\%).La escala adaptativa tuvo mayor presentación para los maestros que para los padres. En opinión de los maestros el 18.3\% de los niños presentaban uno o más trastornos adaptativos; para los padres fue 4.9\%. Similarmente, los maestros reportaron que $9.9 \%$ de los niños presentaban uno o más trastornos en la escala clínica; los padres reportaron $3.6 \%$.

Respecto a la comorbilidad de trastornos, los padres reportaron que el $21.4 \%$ presentaba un trastorno, el $8.4 \%$ presentaba dos trastornos, y el $2.3 \%$ presentaba tres o más trastornos; el $67.9 \%$ de la muestra no presentó ningún trastorno. De acuerdo a los maestros, el 18.3\% presentaba un trastorno, el $14.5 \%$ presentaba dos trastornos, y el 25.2\% presentaba tres o más trastornos; el $42.0 \%$ de la muestra no presentó ningún trastorno. 
Invest. pens. crit.

Vol. 2, No. 3, enero-abril 2014.

pp. $35-52$

Cuando se compara la prevalencia de trastornos entre niños y niñas, no hubo diferencias significativas según los padres (ver Tabla 4). Por otro lado, los maestros consideraban que el aislamiento es más prevalente en niños que en niñas, y reportaron una tasa mayor de problemas de aprendizaje y ansiedad en niñas que en niños.

\section{Discusión}

El presente estudio evaluó la prevalencia de problemas de conducta y emocionales en una muestra de niños de edad escolar en Panamá. La prevalencia total de los trastornos del BASC fue del 8.1\%. Menos de 1 en 10 niños clasificados con al menos un trastorno recibió atención en servicios de salud mental y ningún niño había sido medicado con un fármaco neuropsiquiátrico por un período prolongado.

Los datos del estudio muestran una prevalencia de trastornos dentro del límite inferior a las cifras reportadas en estudios previos realizados en países Latinoamericanos. En Brasil se reportó una prevalencia de $12.7 \%$ en niños entre 7 a 14 años (37). En Puerto Rico se observó una prevalencia de trastornos psiquiátricos en niños de 6.9\% (38). En Panamá no contamos con estadísticas estimadas sobre la prevalencia en trastornos psiquiátricos en niños. Solo recientemente se publicó un estimado de la prevalencia del trastorno por déficit de atención e hiperactividad de 7.4\% (39), cifra que se encuentra dentro del rango de hiperactividad y problemas de atención obtenidas con el BASC en el presente estudio. Si se comparan las puntuaciones $T$ en el BASC para padres obtenidas en una muestra de niños entre 6 y 11 años de estrato socioeconómico bajo de Medellín, Colombia $(n=59)$ (33), la muestra del presente estudio obtuvo puntuaciones más cerca de lo normal (i.e. promedio 50) que la muestra similar de Medellín, lo que se traduce en menores tasas de prevalencia en la muestra de Panamá. En resumen, considerando que aproximadamente $30 \%$ de la población en países latinoamericanos consiste de niños menores de 15 años, aun las cifras de prevalencia más conservadoras (6-7\%) representan un número significativo de niños con problemas de salud mental (23). No obstante, la variabilidad entre nuestras estimaciones y las de otros estudios resalta la necesidad de aclarar el sistema de clasificación de trastornos en niños (18).

En el presente estudio, una falta de acuerdo surgió entre las evaluaciones de los maestros y los padres, evidenciada por la baja correlación entre las puntuaciones $T$ en todas las variables del BASC y en las prevalencias estimadas. En general, la prevalencia de problemas fue mayor en los reportes de los maestros que los padres. Las dificultades en las habilidades para el estudio y los problemas de aprendizaje fueron los trastornos más prevalentes de acuerdo a los maestros; uno de cada cuatro participantes en el estudio tuvo déficits en los hábitos de estudio según los maestros. Además, las diferencias de presencia de problemas conductuales y emocionales entre sexos fueron únicamente encontradas a partir de las evaluaciones de maestros: las niñas evaluadas significativamente más ansiosas y con problemas de aprendizaje que los niños. Mientras 
Invest. pens. crit.

Vol. 2, No. 3, enero-abril 2014.

pp. $35-52$

que los niños fueron evaluados con significativamente más problemas de aislamiento que las niñas.

La mayor discrepancia entre los padres y maestros se observó en la prevalencia de los trastornos de externalización que según los padres se presentaba con una frecuencia mucho menor. Conjuntamente, la correlación significativa entre padres y maestros en las puntuaciones en hiperactividad, agresión y problemas de conducta, y la diferencia significativa en la prevalencia de estas condiciones, indica que los informantes están de acuerdo en cuanto a la presencia pero no la magnitud de estos problemas. En general, los padres y maestros estuvieron de acuerdo en la frecuencia de problemas de ansiedad, depresión y somatización. Estudios realizados sobre la epidemiología de problemas de internalización en niños y adolescentes revelan que estos trastornos tienden a aumentar con la edad, en las mujeres, y en niveles socioeconómicos bajos (40). Además, los trastornos de internalización son los más prevalentes y crónicos, y tienden repercutir de manera negativa sobre la salud mental en la adultez $(5,41)$, lo que resalta la importancia de la intervención temprana (42).

El presente estudio resalta además un tema muy discutido en la literatura de la psiquiatría pediátrica, la del grado de acuerdo entre diferentes informantes en escalas de evaluación conductual. Un estudio meta-analítico de 119 estudios en los que múltiples informantes evaluaron los problemas conductuales y emocionales en niños entre las edades de 1.5 y 19 años (43) encontró que la correlación media entre padres y maestros fue de .27, un valor por arriba de todas las correlaciones reportadas en el presente estudio. La discordancia entre las evaluaciones de los padres y maestros fue evidente en la baja correlación en las puntuaciones $T$, y también en el grado de diferencia en las tasas de prevalencia de las variables del BASC. En estudios anteriores, la prevalencia de problemas de conducta fue menor en evaluaciones de los maestros que de los padres (44-46), lo que se evidenció en dirección opuesta en el presente estudio. Sin embargo, hubo más concordancia entre padres y maestros en cuanto a las conductas de externalización (i.e. hiperactividad, agresión y problemas de conducta) comparado a las conductas de internalización (i.e. depresión, ansiedad), lo que coincide con estudios anteriores $(44,45)$. Revisiones del tema sugieren que el bajo grado de acuerdo entre informantes no se debe a la fiabilidad de las escalas sino a las diferencias contextuales de las conductas evaluadas. Por esta razón existe un consenso que la evaluación psicológica de niños debe tomar esta variación contextual en consideración $(28,29)$.

Una posible explicación en cuanto a las diferencias entre las evaluaciones de padres y maestros es que los maestros tienen expectativas de la conducta de sus alumnos que superan las de los padres. Por otro lado, es posible también que los niños se comporten de manera significativamente diferente en el colegio y el hogar. Estos supuestos deben ser probados de manera explícita en estudios de observación en diferentes entornos. Es probable que las prevalencias reportadas en este estudio sean subestimaciones de las tasas reales en la población debido a que un porcentaje importante de evaluaciones de maestros con índices aceptables no fueron incluidas 
Invest. pens. crit.

Vol. 2, No. 3, enero-abril 2014.

pp. $35-52$

en los estimados de prevalencia. Además, las tasas fueron basadas en evaluaciones de la frecuencia de conductas por parte del padre y el maestro, y no por profesionales de salud mental, por lo que no hubo evaluaciones posteriores que pudieran elaborar los resultados obtenidos con el BASC.

El presente estudio tuvo varias limitaciones. Primero, la selección de dos colegios municipales representa una muestra de conveniencia. Segundo, debido a que la muestra consistió de niños normales de edad escolar entre las edades de 6 y 11 años, la extrapolación de los resultados a niños con problemas conductuales/emocionales referidos a evaluación psicológica o a niños de mayor o menor edad requiere verificación por medio de estudios adicionales. Es evidente que para abordar los problemas de salud mental en la población pediátrica es necesario realizar estudios con grandes muestras de diferentes grupos socioeconómicos. Sin embargo, a pesar de las limitaciones metodológicas, consideramos que el presente estudio es un primer paso hacia conocer la prevalencia de trastornos conductuales y emocionales en niños de edad escolar en Panamá. Los resultados obtenidos sustentan la necesidad de incrementar los esfuerzos orientados a conocer el estatus de la salud mental en la población en general.

En Panamá hay más de 300000 niños entre las edades de 6 y 11 años (30). En base a los estimados de prevalencia, más de 20000 tiene por lo menos un trastorno mental y menos de uno en 10 está recibiendo atención, lo que pudiera repercutir en el desempeño académico y social del individuo. Son necesarios más estudios en diferentes áreas de la geografía nacional para contar con estadísticas locales que permitan mejorar las bases de datos para los trastornos psiquiátricos en niños y adolescentes. Es importante que sigamos cumpliendo con los esfuerzos internacionales para conocer los retos en materia de salud mental que se presentan en nuestro país. Con esta información podemos proponer cambios e innovaciones que nos sitúen en un mejor nivel de comprensión y solución de un problema que afecta, de diferentes maneras, a un alto porcentaje de la población pediátrica.

\section{Agradecimientos}

El estudio fue financiado por la Secretaría Nacional de Ciencia, Tecnología e Innovación (SENACY'T) de Panamá (FID-08-065).

\section{Referencias}

1. De la Barra, F. Epidemiología de los trastornos psiquiátricos en niños y adolescentes: estudios de prevalencia. Rev Chil Neuro-Psiquiat. 2009; 47 (4) 303-14.

2. Merikangas KR, He JP, Brody D, Fisher PW, Bourdon K, Koretz DS. Prevalence and treatment of mental disorders among US children in the 2001-2004 NHANES. Pediatr. 2010; 125(1):75-81. 
Invest. pens. crit.

Vol. 2, No. 3, enero-abril 2014.

pp. $35-52$

3. Remschmidt H, Belfer M. Mental health care for children and adolescents worldwide: a review. World Psychiatry. 2005; 4(3):147-53.

4. Goodman A, Joyce R, Smith JP. The long shadow cast by childhood physical and mental problems on adult life. Proc Natl Acad Sci USA. 2011; 108(15): 6032-7.

5. Harrington R, Clarke, A. Prevention and early intervention for depression in adolescence and early adult life. Euro Arch Psy Clin N. 1998; 248(1): 32-45.

6. Organización Panamericana de la Salud. Estrategias y Plan de Acción para la Salud Mental. Washington DC; 2009.

7. Campbell P. A decade for psychiatric disorders. Nature.2010; 463:9.

8. Organización Panamericana de la Salud. Declaración de Caracas; 1990.

9. Organización Panamericana de Salud. Conferencia OPS Panamá 2010. Disponible en: http://new.paho.org/hq. Consultado 5 Noviembre 2010.

10. Andersen S. Trajectories of brain development point of vulnerability or window of opportunity. Neurosci Biobehav Res. 2003; 27 (1-2): 3- 18.

11. Spear L. The adolescent brain and age-related behavioral manifestations. Neurosci Biobehav Res. 2000; 24 (4): 417-63.

12. Evans GW, Schamberg MA. Childhood poverty, chronic stress and adult working memory. Proc Natl Acad Sci USA. 2009; 106(16): 6545-49.

13. Hackman D, Farah M, Meaney M. Socioeconomic status and the brain mechanistic insights from human and animal research. Nat Rev Neurosci; 2010; 11: 651- 59

14. Belfer ML, Rohde LA. Child and adolescent mental health in Latin America and the Caribbean: problems, progress, and policy research. Rev Panam Salud Pública. 2005; 18(4-5): 359-65.

15. World Health Organization. Atlas: child and adolescent mental health resources: global concern: implications for the future. Geneva: WHO; 2005.

16. Organización Panamericana de la Salud. Clasificación Estadística Internacional de Enfermedades y Problemas Relacionados con la Salud Décima revisión. Washington DC: OPS; 1995 
Invest. pens. crit.

Vol. 2, No. 3, enero-abril 2014.

pp. $35-52$

17. American Psychiatric Association. Diagnostic and Statistical Manual of Mental Disorders 4th. ed. Washington: APA; 1994.

18. Mash EJ, Hunsley J. Evidence-based assessment of child and adolescent disorders: issues and challenges. J Clin Child Adolesc Psychol. 2005; 34(3):362-79.

19. Roberts R E, Atkinson C, Rosenblatt A. Prevalence of psychopathology among children and adolescents. Amer J Psychiatry. 1998; 155: 715-25.

20. Levitt J M, Saka N, Romanelli L H, Hoagwood K. Early identification of mental health problems in schools: the status of instrumentation. J School Psychol. 2007; 45(2): 163-91.

21. Nikapota A. Child psychiatry in development countries. Brit J Psychiatry. 1991; 158: 743-51.

22. Patel V, Flisher A J, Hetrick S, et al. Mental health of young people: a global public health challenge. Lancet 2007; 369: 1302-13.

23. Duarte C. Child mental health in Latin American: present and future epidemiologic research. Int J Psychiat Med. 2003; 33(3): 203-22.

24. Organización Mundial de la Salud. Informe sobre salud mental en el mundo 2001, salud mental: nuevos conocimientos, nuevas esperanzas. Ginebra; 2001.

25. World Health Organization. The global burden of disease: 2004 update; 2008.

26. Kohn R, Levav I, Caldas JM, Vicente B, Andrade L, Caraveo-Anduaga J, et al. Los trastornos mentales en América Latina y el Caribe: asunto prioritario para la salud pública. Rev Panam Salud Pública. 2005; 18(4/5): 229-40.

27. Guralnick MJ. The effectiveness of early intervention. Baltimore: Brookes; 1997.

28. Holmbeck GN, Thill AW, Bachanas P, Garber J, Miller KB, Abad M, et al. Evidencebased assessment in pediatric psychology: measures of psychosocial adjustment and psychopathology. J Pediatr Psychol. 2008; 33(9):958-80.

29. Myers K, Winters NC. Ten-year review of rating scales. I: overview of scale functioning, psychometric properties, and selection. J Am Acad Child Adolesc Psychiatry. 2002; 41: 114-22.

30. Instituto Nacional de Estadística y Censo. Contraloría General de la República. Censos Nacionales de Población y Vivienda, Panamá, República de Panamá; 2000. 
Invest. pens. crit.

Vol. 2, No. 3, enero-abril 2014.

pp. $35-52$

31. Reynolds C, Kamphaus R. The Clinician's guide to the behavior assessment system for children. New York: Gilford Press; 2002.

32. Pineda DA, Henao GC, Puerta IC, Mejía SE, Gómez LF, Miranda ML, et al. Uso de una escala multidimensional para padres de niños de 6 a 11 años en el diagnóstico de deficiencia atencional con hiperactividad. Rev Neurol. 1999; 28(10): 952-9.

33. Pineda DA, Kamphaus RW, Mora O, Restrepo MA, Puerta IC, Palacio LG, et al. Sistema de evaluación multidimensional de la conducta. Escala para padres de niños de 6 a 11 años, versión colombiana. Rev Neurol. 1999; 28(6): 672-81.

34. McCloskey D, Hess R, D'Amato. Evaluating the Utility of the Spanish Version of the Behavior Assessment System for Children-Parent Report System. J Psychoeduc Assess. 2003; 21: 325-35.

35. Puerta IC. Instrumentos para evaluar las alteraciones de la conducta. Rev Neurol. 2004; 38(3): 271-7.

36. Rescorla L, Achenbach TM, Almqvist F, Bird H, Broberg A, Dobrean A, et al. Epidemiological comparisons of problems and positive qualities reported by adolescents in 24 countries. J Consult Clin Psych. 2007; 75:351-8.

37. Fleitlich-Bilyk B, Goodman R. The prevalence of child psychiatric disorders in South East Brazil. J Am Acad Child Adolesc Psychiatry 2004; 43:727-34.

38. Canino G, Shrout P, Rubio-Stipec M, et al. The DSM-IV rates of child and adolescent disorders in Puerto Rico. Arch Gen Psychiatry 2004; 61: 85-93.

39. Sánchez EY, Velarde S, Britton GB. Estimated prevalence of attentiondeficit/hyperactivity disorder in a sample of Panamanian school-aged children. Child Psychiatry Hum Dev. 2011; 42(2): 243-55.

40. Birmaher B, Ryan ND, Williamson DE, Brent DA, Kaufman J, Dahl RE, et al. Childhood and adolescent depression. I: a review of the past 10 years. J Am Acad Child Adolesc Psychiatry. 1996; 35:1427-39.

41. Kasen S, Cohen P, Skodol AE, Johnson JG, Smailes E, Brook JS. Childhood depression and adult personality disorder: alternative pathways of continuity. Arch Gen Psychiatry. 2001; 58(3):231-6.

42. Cuijpers P, van Straten A, Smits N, Smit F. Screening and early psychological intervention for depression in schools. Eur Child Adolesc Psychiatry. 2006; 15:300-7. 
Invest. pens. crit.

Vol. 2, No. 3, enero-abril 2014.

pp. $35-52$

43. Achenbach TM, McConaughy SH, Howell CT. Child/adolescent behavioral and emotional problems: implications of cross-informant correlations for situational specificity. Psychol Bull. 1987; 101:213-32.

44. Cai X, Kaiser AP, Hancock T. Parent and teacher agreement on child behavior checklist items in a sample of preschoolers from low-income and predominantly African American families. J Clin Child Adolesc Psychiatry. 2004; 33(2): 303-12.

45. Flanagan DP, Alfonso VC, Primavera LH, Poval L, Higgins D. Convergent validity of the BASC and SSRS: implications for social skills assessment. Psychol Schools. 1996; 33:13-23.

46. Loeber R, Green SM, Lahey BB, Stouthamer-Loeber R. Differences and similarities between children, mothers, and teachers as informants on disruptive child behavior. $J$ Abnorm Child Psychol. 1991; 19:75-95. 
Invest. pens. crit.

Vol. 2, No. 3, enero-abril 2014.

pp. 35-52

\section{Tablas}

Tabla 1. Características de la muestra

\begin{tabular}{|c|c|c|c|}
\hline & & \multicolumn{2}{|c|}{ Valores } \\
\hline & & (n) & $(\%)$ \\
\hline \multirow[t]{2}{*}{ Sexo } & Femenino & 71 & 54.2 \\
\hline & Masculino & 60 & 45.8 \\
\hline \multirow[t]{6}{*}{ Edad } & 6 & 24 & 18.3 \\
\hline & 7 & 34 & 26.0 \\
\hline & 8 & 32 & 24.4 \\
\hline & 9 & 17 & 13.0 \\
\hline & 10 & 21 & 16.0 \\
\hline & 11 & 3 & 2.3 \\
\hline \multirow[t]{3}{*}{ Educación de la madre } & Primaria & 12 & 9.2 \\
\hline & Secundaria & 82 & 62.6 \\
\hline & Universidad & 27 & 20.6 \\
\hline \multirow[t]{3}{*}{ Educación del padre } & Primaria & 17 & 13.0 \\
\hline & Secundaria & 88 & 67.2 \\
\hline & Universidad & 18 & 13.7 \\
\hline \multirow[t]{2}{*}{ Padres juntos } & Sí & 86 & 65.7 \\
\hline & No & 43 & 32.9 \\
\hline \multirow[t]{2}{*}{$\begin{array}{l}\text { Acudió a servicios de salud } \\
\text { mental }\end{array}$} & Sí & 15 & 11.5 \\
\hline & No & 116 & 88.6 \\
\hline \multirow{2}{*}{$\begin{array}{l}\text { Recibió fármacos } \\
\text { neuropsiquiátricos por más de } 6 \\
\text { meses }\end{array}$} & Sí & 0 & 0 \\
\hline & No & 131 & $100 \%$ \\
\hline
\end{tabular}


Invest. pens. crit.

Vol. 2, No. 3, enero-abril 2014.

pp. $35-52$

Tabla 2. Puntuaciones estandarizadas T en las variables del BASC para padres y maestros de 131 niños en la ciudad de Panamá.

\begin{tabular}{|c|c|c|c|c|c|}
\hline \multirow[b]{2}{*}{ Variables } & \multicolumn{2}{|c|}{ Padres } & \multicolumn{2}{|c|}{ Maestros } & \multirow[b]{3}{*}{$r^{\mathrm{a}}$} \\
\hline & Promedio & $\mathrm{DE}$ & Promedio & $\mathrm{DE}$ & \\
\hline Escala clínica & & & & & \\
\hline Hiperactividad & 44.85 & 7.13 & 48.90 & 8.72 & $.116^{*}$ \\
\hline Agresión & 45.22 & 6.91 & 46.68 & 10.03 & $.157^{*}$ \\
\hline Problemas de conducta & 44.28 & 7.88 & 49.86 & 8.29 & $.171 *$ \\
\hline Ansiedad & 48.57 & 9.24 & 49.15 & 10.12 & .037 \\
\hline Depresión & 44.38 & 8.06 & 45.66 & 9.77 & .031 \\
\hline Somatización & 48.26 & 7.41 & 46.14 & 7.92 & .058 \\
\hline Atipicidad & 47.69 & 7.04 & 49.69 & 7.90 & .050 \\
\hline Aislamiento & 50.48 & 7.70 & 46.05 & 10.04 & .069 \\
\hline Problemas de atención & 45.33 & 9.20 & 48.93 & 9.35 & $.219 * *$ \\
\hline Problemas de aprendizaje & N.A. & N.A. & 49.95 & 10.53 & N.A. \\
\hline \multicolumn{6}{|l|}{ Escala adaptativa } \\
\hline Adaptabilidad & 55.32 & 11.28 & 47.32 & 9.96 & .098 \\
\hline Habilidades sociales & 53.99 & 8.93 & 50.28 & 11.02 & .039 \\
\hline Liderazgo & 50.98 & 7.34 & 51.33 & 9.93 & .047 \\
\hline Habilidades de estudio & N.A. & N.A. & 48.19 & 10.29 & N.A. \\
\hline
\end{tabular}

$\mathrm{DE}=$ desviación estándar

${ }^{a}$ Correlación (coeficiente Pearson) entre padres y maestros $\left({ }^{*} p<.05 ; * * p<.01\right)$ 
Invest. pens. crit.

Vol. 2, No. 3, enero-abril 2014.

pp. 35-52

Tabla 3. Prevalencia estimada para la muestra $(n=131)$ de acuerdo a las puntuaciones T para cada variable del BASC en cuestionarios de padres y maestros.

\begin{tabular}{|c|c|c|c|c|}
\hline \multirow[b]{2}{*}{ Variables } & \multicolumn{2}{|c|}{ Padres } & \multicolumn{2}{|c|}{ Maestros } \\
\hline & Frecuencia & $\% \pm \mathrm{EE}$ & Frecuencia & $\% \pm \mathrm{EE}$ \\
\hline \multicolumn{5}{|l|}{ Escala clínica } \\
\hline Hiperactividad & 1 & $0.8 \pm 0.78$ & 14 & $10.7 \pm 2.70^{*}$ \\
\hline Agresión & 1 & $0.8 \pm 0.78$ & 11 & $8.4 \pm 2.42 *$ \\
\hline Problemas de conducta & 2 & $1.5 \pm 1.06$ & 10 & $7.6 \pm 2.32 *$ \\
\hline Ansiedad & 11 & $8.4 \pm 2.42$ & 20 & $15.3 \pm 3.15$ \\
\hline Depresión & 3 & $2.3 \pm 1.31$ & 9 & $6.9 \pm 2.21$ \\
\hline Somatización & 3 & $2.3 \pm 1.31$ & 6 & $4.6 \pm 1.83$ \\
\hline Atipicidad & 2 & $1.5 \pm 1.06$ & 11 & $8.4 \pm 2.42 *$ \\
\hline Aislamiento & 14 & $10.7 \pm 2.70$ & 12 & $9.2 \pm 2.53$ \\
\hline Problemas de atención & 5 & $3.8 \pm 1.67$ & 9 & $6.9 \pm 2.21$ \\
\hline Problemas de aprendizaje & N.A. & N.A. & 27 & $20.6 \pm 3.53$ \\
\hline \multicolumn{5}{|l|}{ Escala adaptativa } \\
\hline Adaptabilidad & 4 & $3.1 \pm 1.51$ & 31 & $23.7 \pm 3.72 *$ \\
\hline Habilidades sociales & 6 & $4.6 \pm 1.83$ & 18 & $13.7 \pm 3.00^{*}$ \\
\hline Liderazgo & 9 & $6.9 \pm 2.21$ & 13 & $9.9 \pm 2.61$ \\
\hline Habilidades de estudio & N.A. & N.A. & 34 & $26.0 \pm 3.83$ \\
\hline
\end{tabular}

$\mathrm{EE}=$ error estándar

$*$ Diferencia significativa entre padres y maestros $\left(\mathbf{0}^{2}, p<.05\right)$. 
Invest. pens. crit.

Vol. 2, No. 3, enero-abril 2014.

pp. $35-52$

Tabla 4. Prevalencia estimada ${ }^{a}$ según género para la muestra $(n=131)$ de acuerdo a las puntuaciones T para cada variable del BASC en cuestionarios de padres y maestros.

\begin{tabular}{|c|c|c|c|c|}
\hline \multirow[b]{2}{*}{ Variables } & \multicolumn{2}{|c|}{ Padres } & \multicolumn{2}{|c|}{ Maestros } \\
\hline & Femenino & Masculino & Femenino & Masculino \\
\hline \multicolumn{5}{|l|}{ Escala clínica } \\
\hline Hiperactividad & $1(1.4 \%)$ & $0(0 \%)$ & $10(14.1 \%)$ & $4(6.7 \%)$ \\
\hline Agresión & $1(1.4 \%)$ & $0(0 \%)$ & $5(7.0 \%)$ & $6(10.0 \%)$ \\
\hline Problemas de conducta & $2(2.8 \%)$ & $0(0 \%)$ & $7(9.9 \%)$ & $3(5.0 \%)$ \\
\hline Ansiedad & $8(11.3 \%)$ & $3(5.0 \%)$ & $17(23.9 \%)$ & $3(5.0 \%)^{\mathrm{b}}$ \\
\hline Depresión & $2(2.8 \%)$ & $1(1.7 \%)$ & $6(8.5 \%)$ & $3(5.0 \%)$ \\
\hline Somatización & $3(4.2 \%)$ & $0(0.0 \%)$ & $4(5.6 \%)$ & $2(3.3 \%)$ \\
\hline Atipicidad & $2(2.8 \%)$ & $0(0.0 \%)$ & $6(8.5 \%)$ & $5(8.3 \%)$ \\
\hline Aislamiento & $11(15.5 \%)$ & $3(5.0 \%)$ & $3\left(4.2^{\%} \%\right)$ & $9(15.0 \%)^{\mathrm{b}}$ \\
\hline Problemas de atención & $2(2.8 \%)$ & $3(5.0 \%)$ & $6(8.5 \%)$ & $3(5.0 \%)$ \\
\hline Problemas de aprendizaje & N.A. & N.A. & $19(26.8 \%)$ & $8(13.3 \%)^{b}$ \\
\hline \multicolumn{5}{|l|}{ Escala adaptativa } \\
\hline Adaptabilidad & $2(2.8 \%)$ & $2(3.3 \%)$ & $17(23.9 \%)$ & $14(23.3 \%)$ \\
\hline Habilidades sociales & $2(2.8 \%)$ & $4(6.7 \%)$ & $11(15.5 \%)$ & $7(11.7 \%)$ \\
\hline Liderazgo & $6(8.5 \%)$ & $3(5.0 \%)$ & $5(7.0 \%)$ & $8(13.3 \%)$ \\
\hline Habilidades de estudio & N.A. & N.A. & $22(31.0 \%)$ & $12(20.0 \%)$ \\
\hline
\end{tabular}

${ }^{\mathrm{a}}$ Datos reflejan la frecuencia y el porcentaje según género (femenino, $n=71$; masculino, $n=60$ ).

bDiferencia significativa entre sexos según maestros $\left(\chi^{2}, p<.05\right)$. 